\title{
Potential injurious effects of the fine particulate PM2.5 on the progression of atherosclerosis in apoE-deficient mice by activating platelets and leukocytes
}

\author{
Xuecan Zhu ${ }^{1}$, Pei Zhao ${ }^{2}$, Yonggang $\mathrm{Lu}^{2}$, Lijing Huo ${ }^{2}$, Mingchen Bai ${ }^{2}$, Fang $\mathrm{Yu}^{2}$, Yanqing Tie ${ }^{1,2}$
}

${ }^{1}$ Hebei North University, Hebei, China

${ }^{2}$ Clinical Laboratory, Hebei General Hospital, Shijiazhuang, Hebei, China

Submitted: 16 January 2018

Accepted: 30 May 2018

Arch Med Sci 2019; 15, 1: 250-261

DOI: https://doi.org/10.5114/aoms.2018.81039

Copyright @ 2018 Termedia \& Banach

\section{Abstract}

Introduction: Exposure to the fine particulate matter PM2.5 is strongly associated with atherosclerotic diseases, creating considerable public concern. Nevertheless, the mechanisms have not been fully elucidated. We exposed atherosclerosis-prone apoE-deficient mice to PM2.5 to begin investigating these mechanisms.

Material and methods: Thirty-two 8-week-old male apoE $\mathrm{E}^{-/-}$mice were divided to two groups fed with high-fat diet: a control group instilled with $0.9 \%$ saline, and an experimental group instilled with PM2.5 (30 mg/kg/day) for 8 weeks. We measured PM2.5 in whole blood by the ICP-MS method, and lipids and inflammatory factors by standard methods. The whole descending arteries were stained with oil red O; Aortic roots were stained with Movat, Sirius Red and immunohistochemical stains for pathological analysis; Brachiocephalic arteries for scanning electron microscopy, the descending arteries for Q-PCR. Echocardiography was used to evaluate cardiac function. Results: In PM2.5 group, we observed elevated heavy metal components, consistent with higher amounts of platelets in total blood. The PM2.5 group also had elevated serum inflammatory factor levels. Finally, the PM2.5 group showed larger atherosclerotic plaques $(p=0.0231)$, higher numbers of lesion macrophages $(p=0.0183)$, greater injury to endothelial layers with greater adherence of platelets and leukocytes, elevated inflammatory factor levels, the NAD $(P) H$ oxidase subunits p22phox and p47phox $(p=0.0079$ and $p=0.0294)$, the M1/ M2 associated markers IL-6, TNF- $\alpha(p=0.0291, p=0.0286)$, iNOS, IL-12 ( $p=$ 0.0122 and $p=0.0280)$ and arginase -1 , and CD206 ( $p=0.0216$ and $p=0.0317)$. Conclusions: PM2.5 exposure activated circulating leukocytes, platelets and associated inflammatory factors, contributing to the progression of atherosclerosis in $\mathrm{apoE}^{-/-}$mice.

Key words: PM2.5, atherosclerosis, M1, M2 macrophages, inflammation, platelets.

\section{Introduction}

Shijiazhuang, the provincial capital of Hebei province, suffers from serious air pollution, especially with the fine particulate PM2.5, and it is also plagued by cardiovascular diseases. The two phenomena are likely causally related, creating widespread public concern $[1,2]$. Atherosclerosis, the principle pathology of cardiovascular disease, is thought to represent a chronic inflammatory disease. Circulating leukocytes and platelets participate in inflammatory responses that drive atherogenic expansion

\author{
Corresponding author: \\ Prof. Yanqing Tie \\ Clinical Laboratory \\ Hebei General Hospital \\ 348 Heping Road \\ Xinhua District \\ Shijiazhuang 050051, China \\ E-mail: tyq1995@126.com
}


[3]. Until recently, air pollutants had been regarded as risk factors for atherosclerosis, particularly the air contaminant PM2.5. It is thought that PM2.5 evades respiratory system barriers and enters the circulation directly, where it alters the functions of leukocytes, induces oxidative stress, and disrupts innate immunity mechanisms $[4,5]$. Nevertheless, the mechanisms leading to atherosclerosis have not been fully elucidated.

Circulating leukocytes, mainly monocytes and macrophages, are components of the innate immunity system responsible for maintaining physiologic equilibrium and monitoring extraneous materials by inducing inflammatory responses. This mechanism evolved to clear foreign substances $[6,7]$. Under pathological conditions, persistent or uncontrolled inflammation may cause host organ damage and atherosclerosis, leading to myocardial infarction and stroke. The initial phase of atherosclerosis appears to be damage to endothelial barriers at arterial bifurcations under hyperlipidemic conditions [8]. Circulating platelets are recruited immediately and express adhesion factors and receptors, including P-selectin, CD4OL, VI and glycoprotein $\mathrm{Ib}$, directing platelet-leukocyte interaction to facilitate leukocyte accumulation and activation, creating a vicious cycle $[6,7,9]$. Circulating monocytes migrate into lesions and subsequently differentiate into macrophages, the major cellular component in plaques. Heterogeneous alterations in macrophage secretory function determine the progression of atherosclerosis. Generally, macrophages can be divided into two sub-populations: classically activated macrophages (M1) release abundant inflammatory cytokines as well as oxidative stress, causing leukocyte recruitment, activation, differentiation and survival. Most prominent among these inflammatory cytokines are tumor necrosis factor $\alpha$ (TNF- $\alpha$ ), IL-6, IL-1 $\beta$, and type I interferon (IFN- $\alpha, \beta)$; the second sub-population consists of alternatively activated macrophages (M2) that elaborate anti-inflammatory and anti-atherogenic effects $[10,11]$ Therefore, the balance between $\mathrm{M} 1$ and $\mathrm{M} 2$ macrophages is critical for development or inhibition of atherosclerosis. PM2.5 is a newly discovered inducer of atherosclerosis. It presumably promotes oxidative stress and visfatin expression. Its effector molecules are currently under investigation.

\section{Material and methods}

\section{Preparation of animals}

Eight-week-old male apoE ${ }^{-/-}$mice were purchased from Cavens Laboratory Animal Co, Ltd (Changzhou, China). All mice were fed a high-fat and cholesterol-rich atherogenic diet (containing 21\% fat, 19.5\% casein, and $1.25 \%$ cholesterol) for 8 weeks at 20 $24^{\circ} \mathrm{C}$ and $45-55 \%$ humidity with a 12 -h light-dark cycle. The mice were divided randomly into the
PM2.5 group $(n=16)$ and the control group $(n=16)$. Mouse body weight was $20 \pm 2 \mathrm{~g}$. In the PM2.5 group, $10 \mathrm{mg}$ of purified power of PM2.5 was suspended in $4.8 \mathrm{ml}$ of carboxy methyl cellulose sodium (CMC) solution for daily dosages, and every mouse received $0.3 \mathrm{ml}$ of solution by gavage once a day for 8 weeks with a high-fat diet. The control group received the same dosage of CMC. All manipulations of animals conformed with the animal ethics committee of Hebei General Hospital as well as the NIH Guide for the Care and Use of Laboratory Animals.

\section{PM2.5 preparation}

The particulate matter (PM) was provided by Hebei Provincial Center for Disease Control and Prevention in Shijiazhuang, China. The PM2.5 samples were prepared using the PM2.5 air sampler and were collected on membrane filters for 24 h/day. PM2.5 membrane filters were weighed and dissolved in normal saline and were further sonicated for $30 \mathrm{~min}$ to scatter the particles and obtain supernatant fluid according to previous studies [5]. Finally, PM2.5 was frozen by liquid nitrogen, dried, and stored at $4^{\circ} \mathrm{C}$ in the dark [4].

Measurement of serum glucose, lipids, IL-6 and TNF- $\alpha$ levels

Blood removed from the eye of anesthetized mice was collected in procoagulant to obtain serum. Serum glucose levels were examined using the glucose oxidase method (Beckman). Total cholesterol (TC), triglycerides (TG), low-density lipoprotein-cholesterol (LDL-C), high-density lipoprotein-cholesterol (HDL-C), IL- 6 and TNF- $\alpha$ levels were tested by ELISA kits from Neobioscience Diagnostics.

\section{Measurement of whole blood heavy metals}

Ten $\mathrm{mg} / \mathrm{l}$ standard solutions of aluminum, vanadium, iron, manganese, selenium and silver (Agilent Technologies Inc, California, American) were used to formulate various mass concentrations of 0.00 , 5.00, 10.00, 20.00, 50.00, 100.00 and $200.00 \mu \mathrm{g} / \mathrm{l}$ to build a standard curve, and then the whole blood levels of appropriate heavy metals were determined by inductively coupled plasma-mass spectrometry (ICP-MS, Thermo Fisher Scientific, Massachusetts, USA) directly.

\section{Oil Red O staining for the descending aorta}

To evaluate the lipid deposition in whole arteries, the aortas were dissected from the left subclavian artery to the iliac bifurcation, then opened longitudinally and stained with Oil Red O for $30 \mathrm{~min}$ and washed with 75\% alcohol. Aortic images were captured with a Sony DXC-960MD (Sony Corporation, Tokyo, Japan), and the lesion size (red pos- 
itive areas) was quantitatively calculated. Data were analyzed by Image-Pro Plus-6 software (Media Cybernetics, Inc).

\section{Scanning electron microscopy (SEM)}

The brachiocephalic arteries (BCA) were dissected and fixed with electron microscope perfusion fixative (2.5\% glutaraldehyde in Millonig's phosphate buffer) for $72 \mathrm{~h}$ and then dehydrated by increasing grades of ethyl alcohol and subsequently dried with a LPD-100 critical point dryer (S4800; Hitachi Limited, Inc., Tokyo, Japan) for 5 min at $41^{\circ} \mathrm{C}$ and 1,200 psi $\mathrm{CO}_{2}$. The specimens were then coated on aluminum stubs with silver glue and observed by SEM $[12,13]$.

\section{Immunohistochemistry, Masson and Sirius red staining}

Aortic roots were sliced into $5 \mu \mathrm{m}$ sections continuously for pathological evaluation. Masson (SS0236; Xinhua Luyuan Science and Technology Ltd, Beijing, China) and Sirius red (DC0041; Leagene Biotechnology Co., Ltd, Beijing, China) staining was performed according to installation instructions to evaluate plaque areas and collagen separately. Sections for immunohistochemistry were first subjected to microwave retrieval and incubated with corresponding antibodies at $4^{\circ} \mathrm{C}$ overnight and subsequently labeled with horseradish peroxidase (HRP)-conjugated anti-rabbit immunoglobulin G ( $\operatorname{lgG})$ at room temperature for $45 \mathrm{~min}$. Finally, they were visualized by DAB and observed by upright microscopy (Carl Zeiss). Antibodies against $\alpha$-actin (1 : 150 dilution) and galectin-3/MAC-2 (1: 200 dilution) were purchased from Santa Cruz Biotechnology (Santa Cruz, CA, USA).

\section{Q-PCR}

Total RNA from the abdominal aorta was isolated using TRIzol (Invitrogen), then treated with DNase I at $37^{\circ} \mathrm{C}$ for 30 min before reverse transcription using a cDNA archive kit (TaKaRa). The CDNA preparations were tested by real-time PCR based on SYBR Green methods, according to the manufacturer's instructions. The amplification and detection of specific products were performed according to the manufacturer's protocol with the IQ5 system (Bio-Rad). Associated gene primers are shown in the supplement.

\section{Echocardiography and hemodynamic measurements}

Transthoracic echocardiography was performed at the end of our experiment. Briefly, prior to ultrasound imaging, mouse thoraxes were shaved and animals were anesthetized with $1 \%$ isoflurane. We used visual sonic vevo770 (VisualSonics, Toronto, Canada). Thirty $\mathrm{MHz}$ high-frequency head scans and associated linear signal transducers were used. The following parameters were recorded: heart rate, left ventricular end systolic dimension $\left(\right.$ LVES $_{d}$ ), left ventricular end diastolic dimension (LVED $)$, left ventricular ejection fraction (LVEF), fractional shortening (FS), left ventricular end diastolic posterior wall thickness $\left(\right.$ LVPW $_{\mathrm{d}}$ ), and left ventricular end diastolic anterior wall thickness $\left(\right.$ LVAW $_{d}$ ). Images were analyzed during end-diastole and during end-systole.

\section{Statistical analysis}

SPSS 11.0 was used as the scientific analysis software and all values were represented as mean \pm SE of the indicated number of measurements. One-way analysis of variance was used to determine significance, and values of $p<0.05$ were considered statistically significant.

\section{Results}

Blood levels of heavy metal content

in $\mathrm{PM} 2.5$-treated apoE $\mathrm{E}^{-/-}$mice

and composition of heavy metals

of purified PM2.5 powder

PM2.5 particulate matter is derived from the burning and emissions from fossil fuel or industrial production. Most toxic heavy metals float in the air, especially aluminum, iron, chromium and arsenic, the major toxic substances. We found remarkably elevated heavy metal levels in whole blood of the PM2.5 group (Table I), especially aluminum and iron. The accurate heavy metal composition of PM2.5 was also measured (Table II), and the proportions were similar to those of whole blood (Table I). These data indicated that PM2.5 heavy metal particles could enter the circulating system by gavage treatment, suggesting further research pathways.

\section{PM2.5 had no significant effects on serum glucose and lipids, but increased pro-inflammatory factor levels}

There were no significant differences in levels of serum glucose, TC, TG, LDL-C or HDL-C between the PM2.5 and control groups (Figure $1 \mathrm{~A}$ ). However, apoE ${ }^{-/-}$mice treated with PM2.5 showed higher serum levels of pro-inflammatory factors (IL- 6 and TNF- $\alpha$ ) than did controls (Figure $1 \mathrm{~B}$ ). Our results suggested that PM2.5 amplified inflammatory responses independently of serum lipid levels.

\section{PM2.5 deteriorated atherosclerotic status observed by SEM}

Brachiocephalic arteries (BCA) are the most atheroma-prone vessels in apoE $\mathrm{E}^{-/-}$mice. Therefore, we 
Table I. Effect of PM2.5 administration on levels of heavy metal contents in whole blood of apoE $\mathrm{E}^{-/}$mice

\begin{tabular}{|lcccccc|}
\hline Group & Aluminum & Vanadium & Iron & Manganese & Selenium & Silver \\
\hline $\begin{array}{l}\text { Control } \\
(n=8)\end{array}$ & $286.5 \pm 30.36$ & $1.585 \pm 0.1052$ & $292700 \pm 29360$ & $15.99 \pm 1.171$ & $647 \pm 41.76$ & $0.2425 \pm 0.02077$ \\
{$[\mu \mathrm{g} / \mathrm{l}]$} & & & & & \\
\hline $\begin{array}{l}\mathrm{PM} 2.5 \\
(n=8)\end{array}$ & $633.6 \pm 96.68^{\star *}$ & $4.174 \pm 0.2713^{\star *}$ & $539800 \pm 13680^{\star *}$ & $19.74 \pm 2.618$ & $690.5 \pm 62.63$ & $0.1625 \pm 0.02889$ \\
{$[\mu \mathrm{g} / \mathrm{l}]$} & & & & & \\
\hline
\end{tabular}

Table II. Contents of the particulate matter PM2.5

\begin{tabular}{|lcccccccc|}
\hline Content & $\mathrm{V}$ & $\mathrm{Al}$ & $\mathrm{Cr}$ & $\mathrm{Mn}$ & $\mathrm{Fe}$ & $\mathrm{As}$ & $\mathrm{Ni}$ & $\mathrm{Cu}$ \\
\hline $\begin{array}{l}\text { Concentration } \\
{[\mu \mathrm{g} / \mathrm{l}]}\end{array}$ & 0.00216 & 0.27302 & 0.01359 & 0.03611 & 0.44658 & 0.01083 & 0.00772 & 0.05719 \\
\hline Content & $\mathrm{Ag}$ & $\mathrm{Cd}$ & $\mathrm{Sb}$ & $\mathrm{Ba}$ & $\mathrm{Tl}$ & $\mathrm{Pb}$ & $\mathrm{Se}$ & $\mathrm{Mo}$ \\
\hline $\begin{array}{l}\text { Concentration } \\
{[\mu \mathrm{g} / \mathrm{l}]}\end{array}$ & 0.00068 & 0.00265 & 0.00573 & 0.02812 & 0.00107 & 0.08645 & 0.01604 & 0.00339 \\
\hline
\end{tabular}

chose BCA for SEM assessment of atherosclerotic surfaces. Compared with the smooth epidermis of control mice (green arrows in Figure $2 \mathrm{~A}$ ), surfaces were "rougher" (indicated by red arrows in Figure 2D) in the PM2.5 group. In addition, we found more adherent leukocytes in experimental tissues (indicated by blue arrows in Figures $2 \mathrm{~B}$ and E) shown as bulging and distended "balls" appearing on the endothelial surface. Most notably, we observed abundant platelets (indicated by yellow arrows, Figures 2 C, F). In conclusion, increased amounts of adherent leukocytes and platelets on the atheroma surface were found after PM2.5 exposure.

A

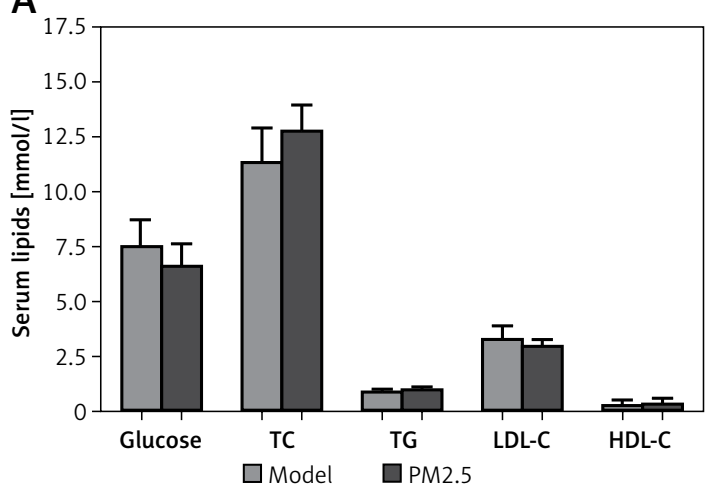

C

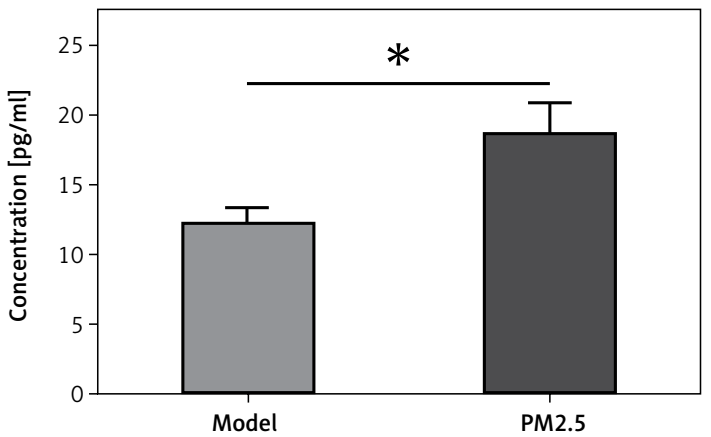

PM2.5 amplified atherosclerotic lesions in apoE $\mathrm{E}^{-/-}$mice

We further evaluated the effect of PM2.5 on the size of atherosclerotic lesions in apoE $\mathrm{E}^{-/-}$mice. Oil Red O stained en face specimens of total descending arteries (initiated from the aortic arch to the bifurcation of the common iliac artery) were used to represent areas occupied by atherosclerotic plaques, measured by the quantitative morphological method. There was a higher percentage of aortic area (lesion area compared to total arch area) in $\mathrm{apoE}^{-/-}$mice treated with PM2.5,

B

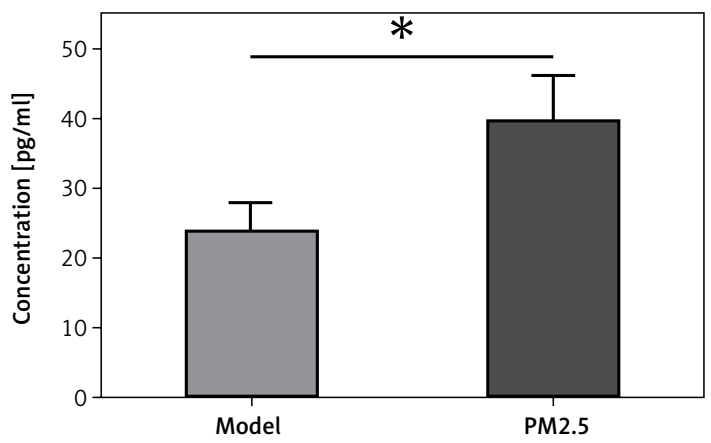

Figure 1. Treatment of apoE $\mathrm{E}^{-/-}$mice with $\mathrm{PM} 2.5$ induced increased serum pro-inflammatory factor levels but had no significant effects on serum glucose and lipids. ApoE ${ }^{-/-}$mice were fed a high-fat, atherogenic diet containing $1.25 \%$ cholesterol and $21 \%$ fat, and were administered PM2.5 (30 mg/kg/ day) for 8 weeks. Serum glucose, TC, TG, LDL-C and HDL-C (A, $p=0.67,0.6915,0.9495,0.5612,0.7851$, respectively), as well as IL- 6 and TNF- $\alpha$ (B, C, $p=0.0196,0.0367)$ were measured by ELISA Data represent the mean $\pm S E(n=8) .{ }^{*} P<0.05$ versus control. 
compared with controls (Figures $3 \mathrm{~A}$ and B). Similarly, we also found increased plaque areas in aortic roots of the PM2.5 group as shown by Masson staining (Figures $4 \mathrm{Aa}$ and $\mathrm{Ba}$ ). These data suggest that PM2.5 treatment facilitated lesion size expansion in $\mathrm{apoE}^{-/-}$mice.

PM2.5 altered lesion cellular contents in apoE $\mathrm{E}^{-/-}$mice

Cellular composition and plaque contents determine the stability of atherosclerotic plaques, particularly macrophages, smooth muscle cells (SMCs) and collagen. To evaluate macrophage and SMC content, we performed immunohistochemical analysis using antibodies against MAC-2 and $\alpha$-actin, respectively. We found increased macrophage numbers in plaques (Figures $4 \mathrm{Ab}$ and $4 \mathrm{Bb}$ ). There was no obvious difference in SMC number with PM2.5 administration (Figures $4 \mathrm{Ac}$ and $4 \mathrm{Bc}$ ), compared with apoE $\mathrm{E}^{-}$mice in the control group. Accordingly, the collagen content was also reduced in plaques, as shown by Sirius red
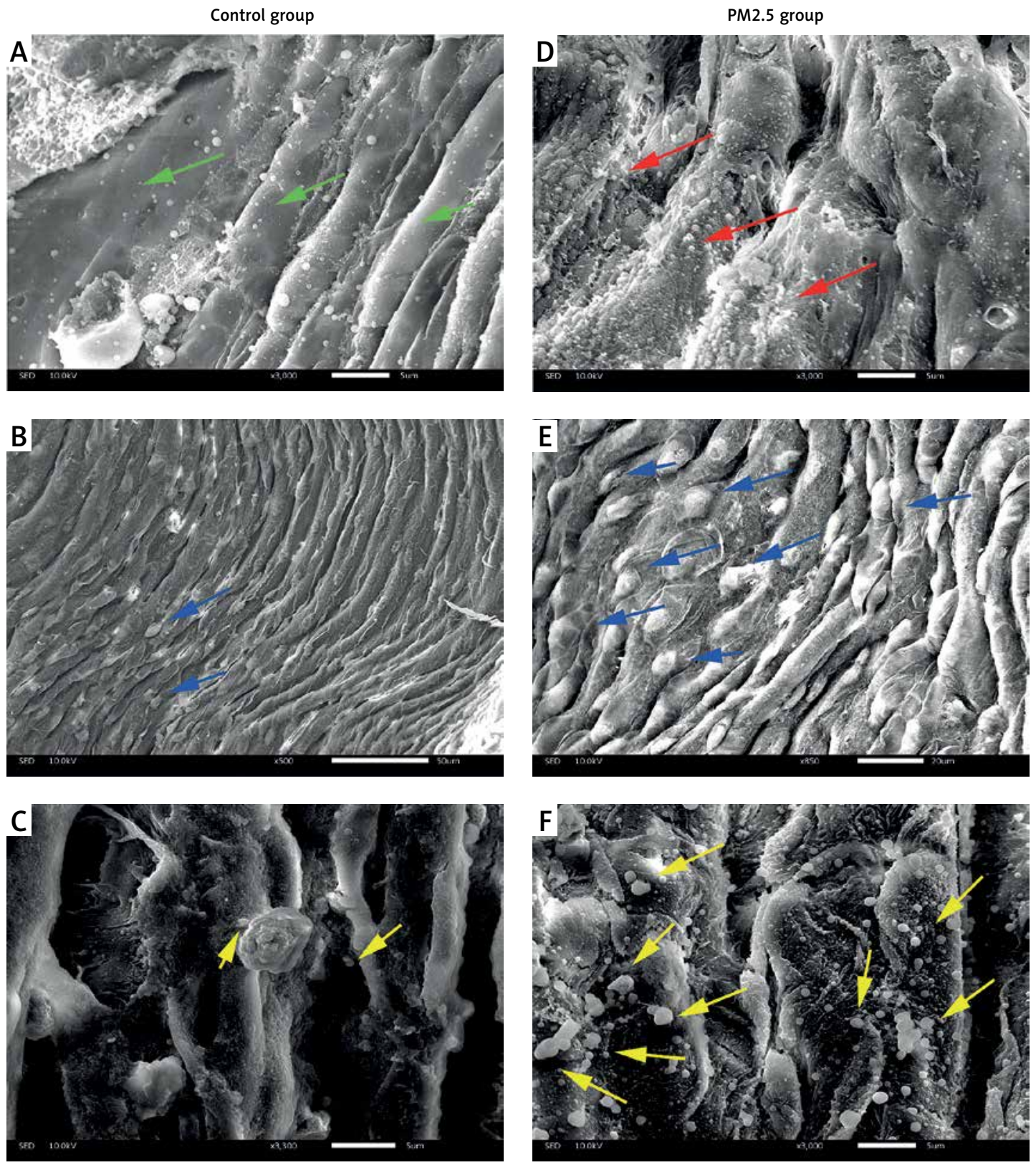

Figure 2. PM2.5 administration induced invasion by leukocytes and platelets, and impaired endothelial surfaces in BCA. Scanning electron microscopy was performed to evaluate the status of the surface of atheroma-prone BCA. More smooth endothelial cells were found in the control (A, green arrows) and rough or damaged surfaces were common in the PM2.5 group (D, red arrows); PM2.5 administration resulted in widespread platelet adherence ( $F$, yellow arrows); adhered leukocytes (E, blue arrows) were found on the surface of arteries 

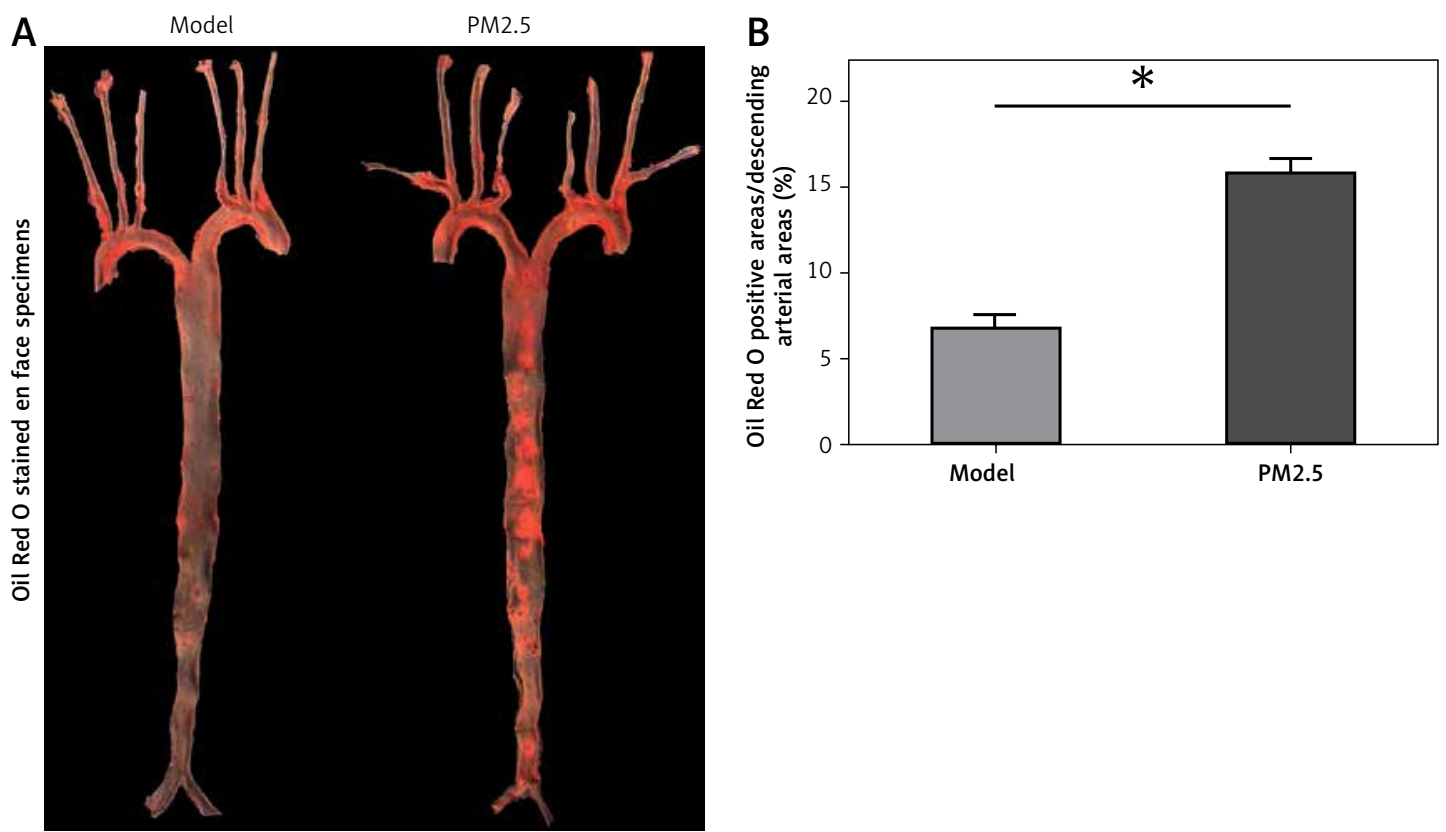

Figure 3. PM2.5 raised areas of atherosclerotic lesions in $a p o E^{-/-}$mice. The ratios of whole aortic lesion areas (Red-positive areas compared to total vessel areas) were measured by quantitative histology of Oil Red O stained en face samples ( $\mathrm{A}$ and $\mathbf{B}, n=8, p=0.0276$ )

Data represent the means $\pm S E .{ }^{*} P<0.05$ versus control.

staining (Figures $4 \mathrm{Ad}$ and $4 \mathrm{Bd}$ ). These results suggest that PM2.5 administration was unfavorable for controlling atherosclerotic stability.

PM2.5 promoted $\mathrm{M} 1$ skewing and release of inflammatory factors by activated macrophages and platelets

Atherosclerosis is a chronic inflammatory disease in which pro-inflammatory factors amplify leukocyte recruitment and inflammatory responses via atherosclerosis-promoting cytokines. Circulating monocytes differentiate into macrophages in atherosclerotic plaques. Macrophages adapt their activation status or phenotype to mount appropriate responses, classified generally as M1 inflammatory and M2 immune regulating subtypes. $M 1$ macrophages synthesize pro-inflammatory factors promoting atherosclerosis progression. M2 macrophages mediate anti-inflammatory and repair functions to inhibit atherosclerosis propagation. We found that classical pro-inflammatory cytokines, IL- 6 and TNF- $\alpha$, were significantly increased, along with increased M1-associated factors, iNOS and IL-12. Simultaneously, we observed decreased M2-specific factors - CD206 and arginase- 1 - in plaques of descending arteries. These data suggested that PM2.5 administration increased inflammatory cytokine release and skewed macrophages toward the M1 phenotype, thus facilitating atherosclerosis progression (Figure $5 \mathrm{Ba}, \mathrm{b}$ and $\mathrm{Ca}, \mathrm{b}$ ).

\section{PM2.5 failed to affect cardiac function as measured by echocardiography}

Quantitative analysis indicated that there were no differences in LVED ${ }_{d}$ and LVES and no differences in LVAW $_{d}$ and LVES $_{d}$ between the groups, suggesting that PM2.5 did not affect the degree of myocardial hypertrophy, and the remaining indices were similar to those of control mice. All data are shown in Figure 6 (Tables III, IV).

\section{Discussion}

We found that PM2.5 exposure accelerated atherosclerotic progression in $\mathrm{apoE}^{-/-}$mice. We found that apoE $E^{-/-}$mice instilled with pure PM2.5 showed: (1) significantly elevated serum pro-inflammatory factors levels, but caused no differences in serum glucose or lipids and failed to affect cardiac function tested by echocardiography; (2) more leukocyte and platelet accumulation on the surface of atheroma observed by SEM; (3) substantial endothelial injury, with greater numbers of platelets and leukocytes adhered; (4) greater lesion areas accompanied by greater numbers of accumulated macrophages, consistent with fewer SMCs and collagen content; and (5) strengthened pro-inflammatory cytokine secretion and lesion-associated macrophages, skewing to the M1 phenotype, with suppression of $\mathrm{M} 2$-associated cytokines as measured by Q-PCR. PM2.5 appeared to accelerate atherosclerotic progression primarily by activating leukocytes and platelets, as well as by activating 
A

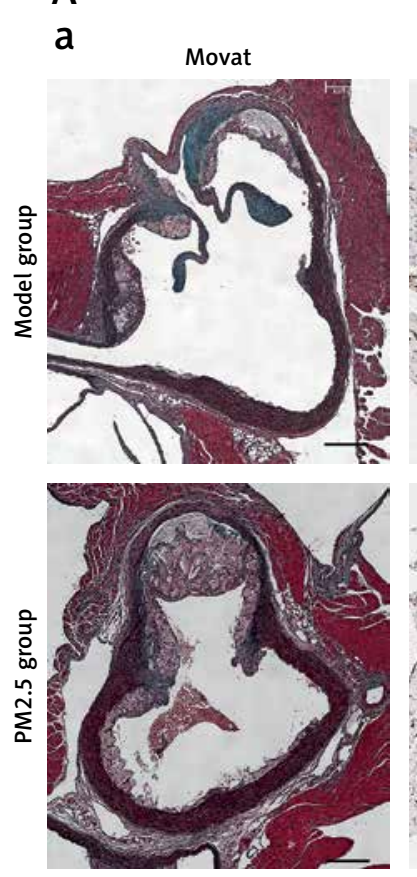

b

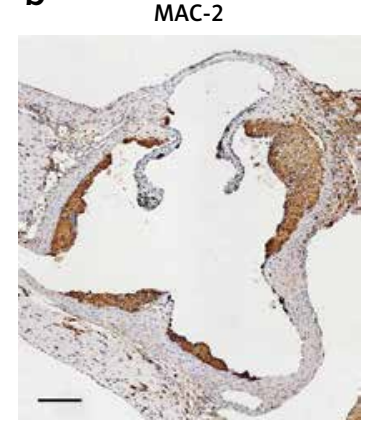

C

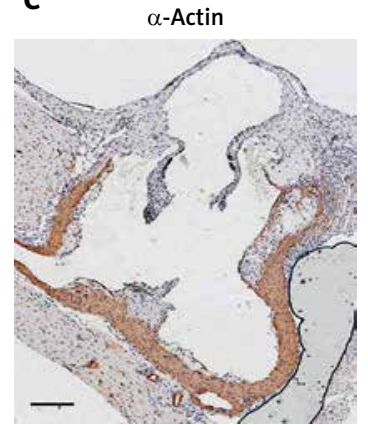

d $\quad$ Sirius red
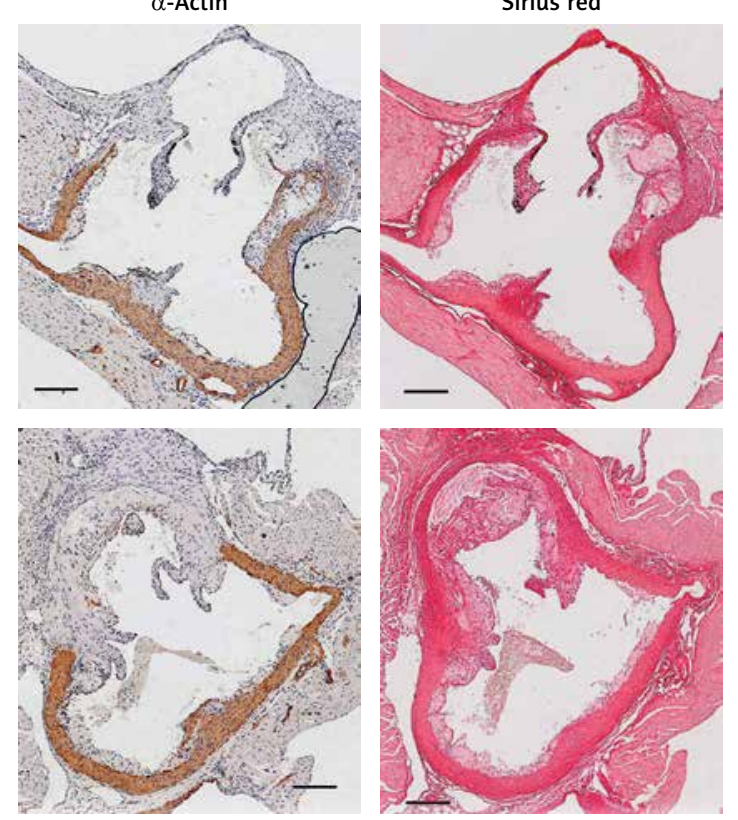

B

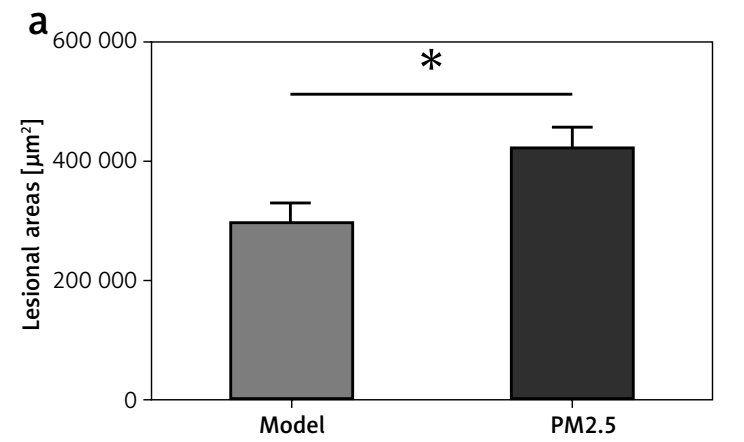

b

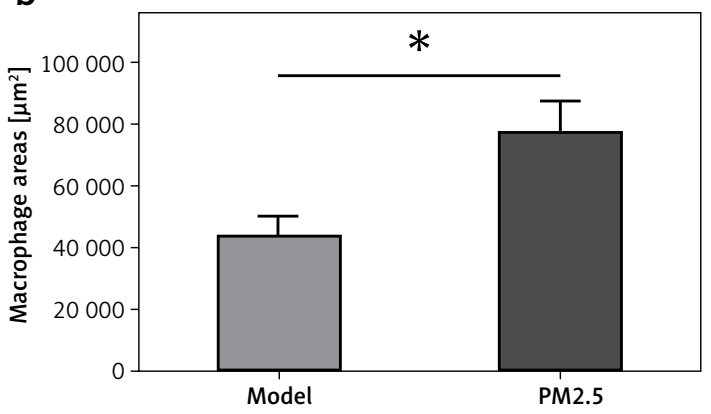

d

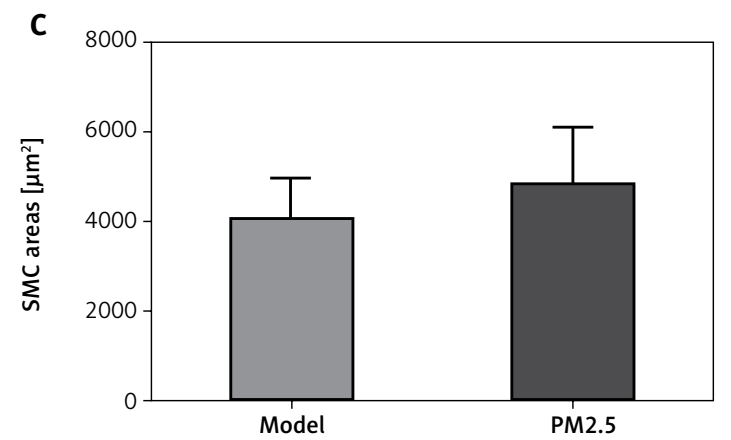

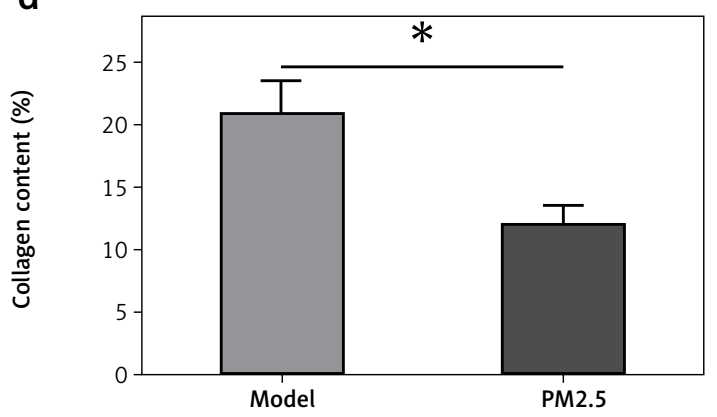

Figure 4. PM2.5 altered lesion areas and cellular composition in aortic roots of apoE $\mathrm{E}^{-/}$mice. Lesion areas in sections from aortic roots were stained with Masson trichrome (Aa, $p=0.0231)$. The quantities of macrophages (Ab, $p=$ 0.0183 ), SMC (Ac, $p=0.6355$ ) and content of collagen (Ad, $p=0.025$ ) in the plaques of aortic roots were determined by immunohistochemistry and Sirius Red staining, respectively

Data represent the means $\pm S E(n=8)$. ${ }^{*} P<0.05$ versus control.

inflammation and oxidative stresses in atherosclerotic plaques by switching macrophages to the $\mathrm{M} 1$ phenotype in apoE $\mathrm{E}^{-/-}$mice.

Much epidemiological and experimental study of the fine particulate PM2.5 suggests that it is a risk factor for atherosclerotic progression, and therefore has acquired considerable attention worldwide. PM2.5 exerts atherogenic effects by inducing endothelial damage, mitochondrial injury, genotoxicity, inflammatory re- 
A

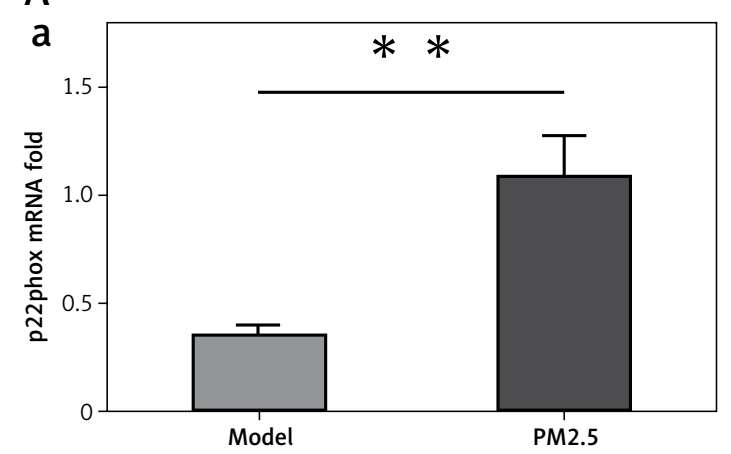

B

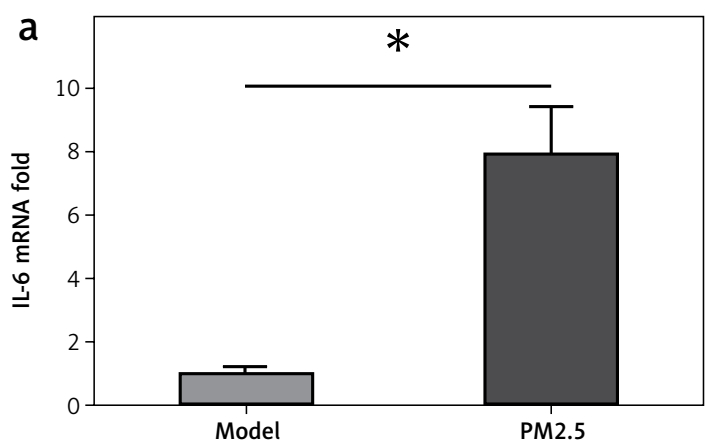

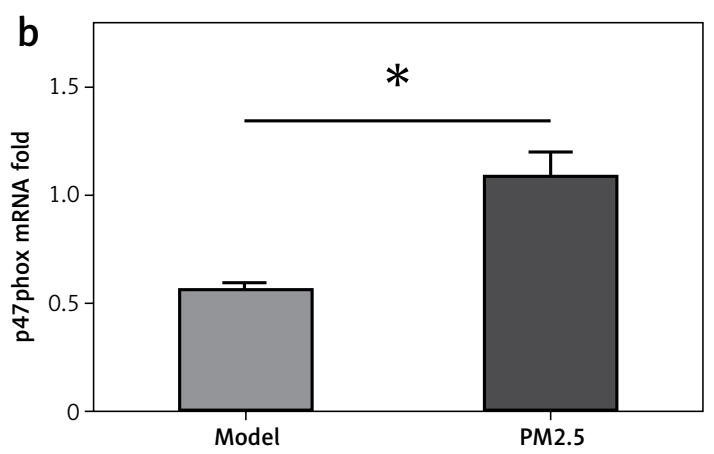

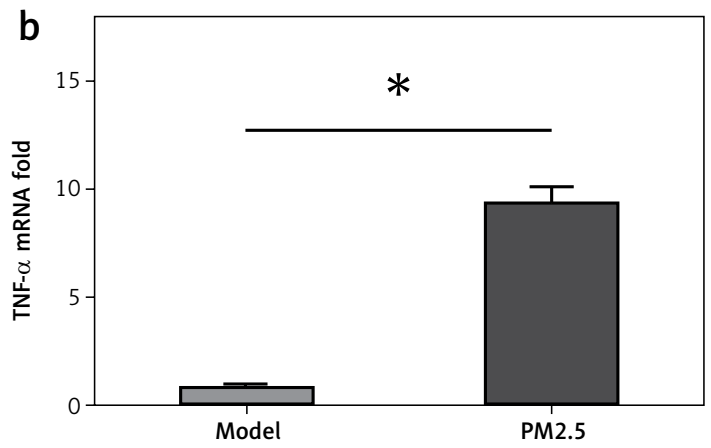

C

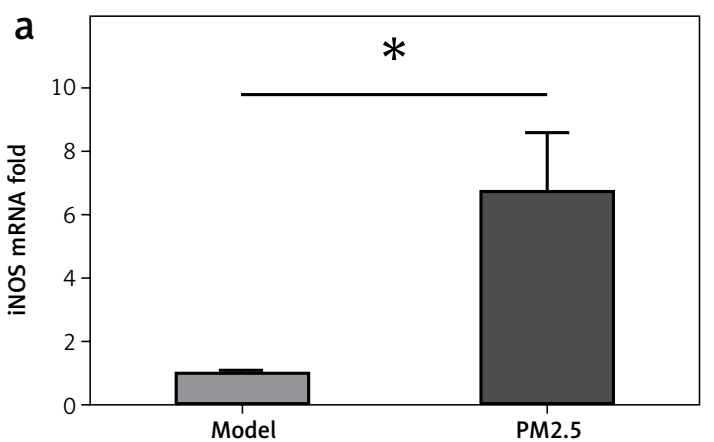

M1-macrophages markers

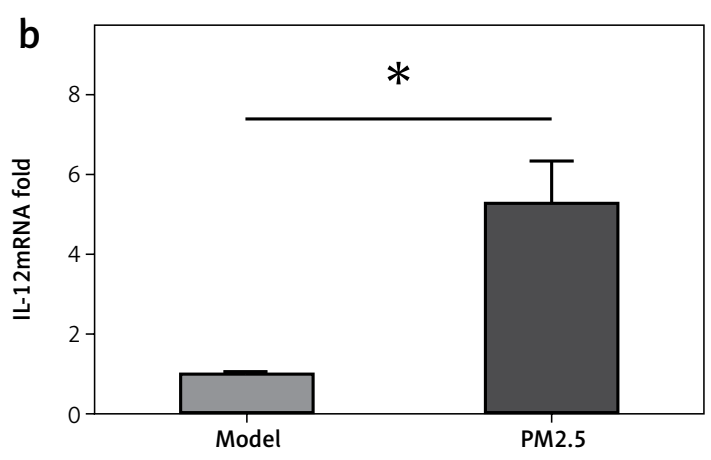

M1-macrophages markers
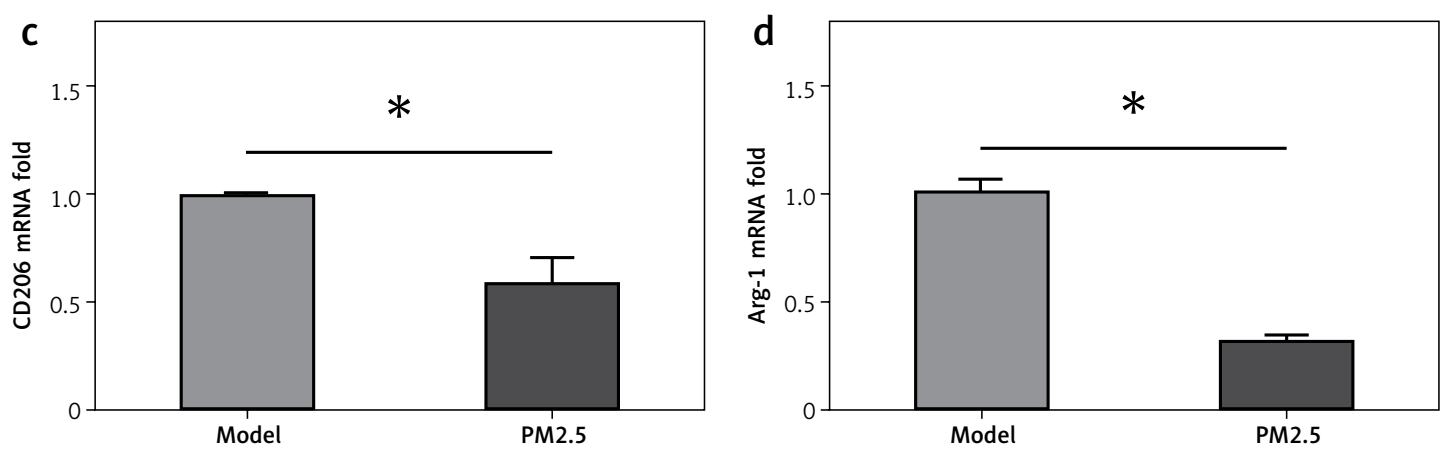

Figure 5. PM2.5 treatment increased the expression of pro-inflammatory factors and M1-associated genes in apoE $\mathrm{E}^{-/-}$mice. $\mathrm{NAD}(\mathrm{P}) \mathrm{H}$ oxidase subunits p22phox and p47phox expression (Aa, b, $p=0.0079$ and 0.0294) and inflammatory cytokines IL-6 and TNF- $\alpha(\mathrm{Ba}, \mathrm{b}, p=0.0291$ and 0.0286$)$ and M1-associated factors iNOS and IL-12 (Ca, b, $p=0.0122$ and 0.0280 ) as well as M2-specific factors CD206 and arginase- 1 (Cc, $\mathbf{d}, p=0.0317$ and 0.0216$)$ from lesions of descending arteries were tested by the Q-PCR method

Data represent the mean $\pm S E .{ }^{*} P<0.05$ versus control. 

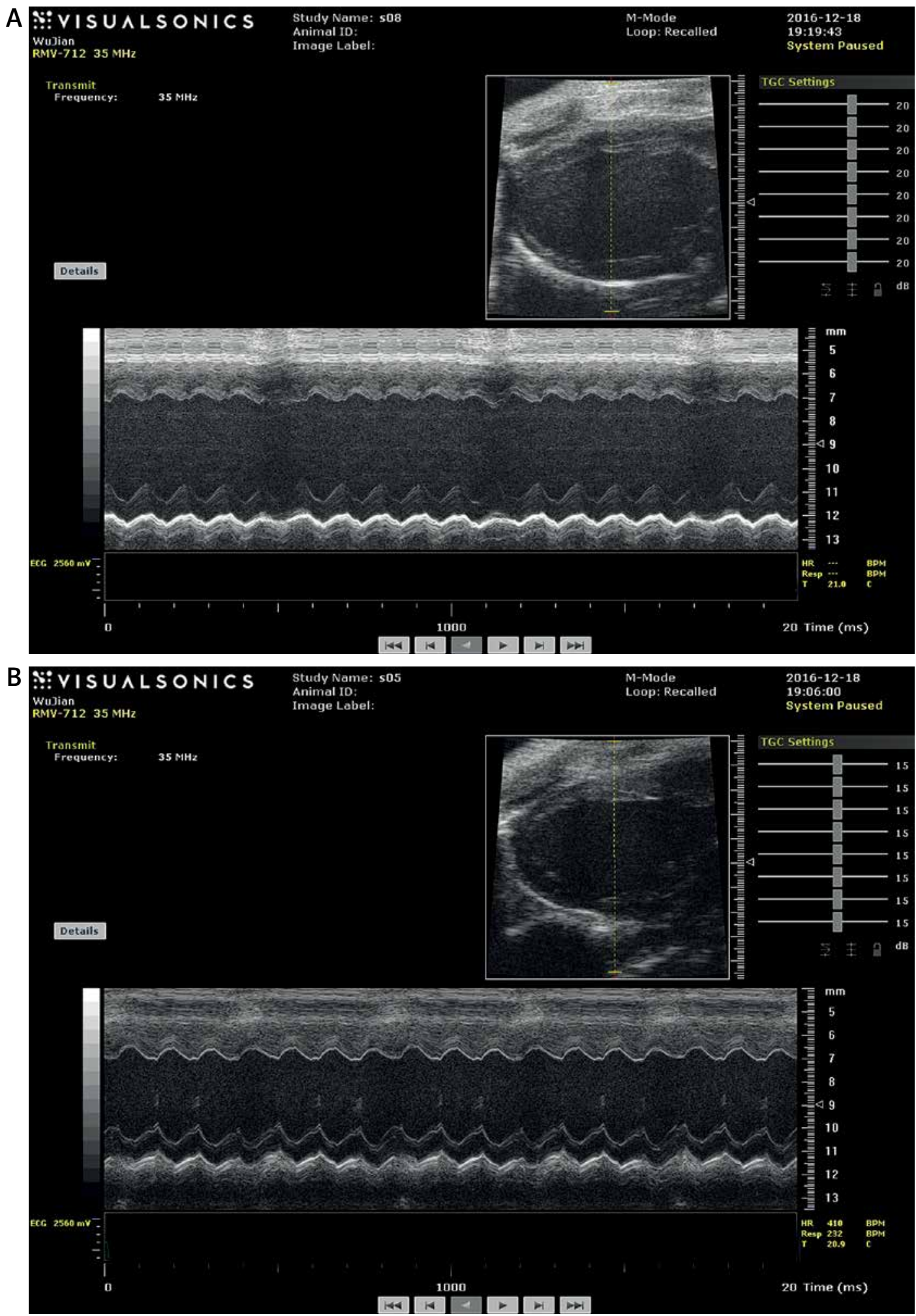

C

\begin{tabular}{|l|c|c|c|c|c|c|}
\hline Group & EF & FS & LVESd & LVEDd & LVPwd & LVAWd \\
\hline Control & $70.54 \pm 7.13$ & $39.84 \pm 4.91$ & $2.48 \pm 0.72$ & $3.81 \pm 0.36$ & $0.74 \pm 0.10$ & $0.79 \pm 0.04$ \\
\hline PM2.5 & $72.74 \pm 9.03$ & $44.19 \pm 8.26$ & $2.52 \pm 0.43$ & $3.89 \pm 0.46$ & $0.82 \pm 0.02$ & $0.85 \pm 0.21$ \\
\hline
\end{tabular}

Figure 6. PM2.5 failed to affect cardiac function tested by echocardiographic images in apoE $\mathrm{E}^{-/-}$mice. $\mathrm{A}-$ Representative control group apoE $\mathrm{E}^{-/-}$mice, heart structure; $\mathrm{B}$ - representative $\mathrm{PM} 2.5$ group, mice exposed to $30 \mathrm{mg} / \mathrm{kg} / \mathrm{day}$, LVAW and LVPW showed no significant difference compared to control mice. Data are shown in Figure $6 \mathrm{C}$ 
sponses and oxidative stress [14]. Furthermore, many toxic compounds are adsorbed to fine particles in air pollutants, especially PM2.5. This agent penetrates the air-blood barrier, thereby damaging the respiratory, cardiovascular, and immune systems. Most developing countries are plagued by both serious atmospheric pollution and atherosclerosis-related diseases; therefore, a new mechanism of this association should be explored.

Atherosclerosis is a chronic inflammatory disease. Atherogenic factors are stimuli that trigger innate immunity and downstream inflammatory reactions. Circulating leukocytes, mainly monocytes and macrophages, are major components of innate immunity that act as the first defense line against foreign bodies [6, 7, 15-17]. Hyperlipidemia and hyperglycemia are classic triggers of atherosclerosis. Nevertheless, we found no significant difference between experimental and control groups with respect to glucose or lipids (Figure 1 A). However, we found elevated TNF- $\alpha$, and IL- 6 levels, both of which are associated with chronic inflammation [18-20]. These data suggest that PM2.5 promoted inflammatory responses in $\mathrm{apoE}^{-/-}$mice under atherosclerotic conditions, independent of an effect on lipid levels. In atherogenic initiation and progression, endothelial barriers break down [8] and platelets are almost immediately recruited. They adhere to endothelia and further express adhesion factors and receptors, including P-selectin, glycoprotein Ib, VI, GPIIb/IIIa and CD40L. The cascade leads to further accumulation and activation of leukocytes, especially monocytes [6, 7, 9]. We found that brachiocephalic arteries (BCA) suffered endothelial damage following PM2.5 treatment. The endothelial surface became rough (Figure $2 \mathrm{D}$, red arrows), with abundant platelets (Figures $2 \mathrm{C}$ and $\mathrm{F}$ yellow arrows), and greater numbers of leukocytes (Figures 2 B, E, blue arrows) adhered on injured surfaces. These data suggested that PM2.5
Table III. Gene primer pairs

\begin{tabular}{|ll|}
\hline TNF- $\alpha-F$ & CAC AGA AAG CAT GAT CCG CG \\
\hline TNF- $\alpha-R$ & ACT GAT GAG AGG GAG GC CAT \\
\hline IL-6-F & AGC CAG AGT CCT TCA GAG AGA \\
\hline IL-6-R & TGG TCT TGG TCC TTA GCC AC \\
\hline iNOS-F & CAG CTG GGC TGT ACA AAC CTT \\
\hline iNOS-R & CAT TGG AAG TGA AGC GTT TCG \\
\hline IL-12-F & GTC TGT GCT GAC CCC AAG AA \\
\hline IL-12-R & AAG GCA TCA CAG TCC GAG TC \\
\hline CD206-F & TTG CAC TTT GAG GGA AGC GA \\
\hline CD206-R & CCT TGC CTG ATG CCA GGT TA \\
\hline Arginase-1-F & :TTT TAG GGT TAC GGC CGG TG \\
\hline Arginase-1-R & CCT CGA GGC TGT CCT TTT GA \\
\hline p22phox-F & CAT CGT GGC TAC TGC TGG AC \\
\hline p22phox-R & TGG ACC CCT TTT TCC TCT TT \\
\hline p47phox-F & CGA AGA AGC CTG AGA CAT ACC \\
\hline p47phox-R & TGTG TCC GTC GTG GAT CTG A CCT TTC CTC ACC ACC \\
\hline GAPDH-F & CCT GCT TCA CCA CCT TCT TGA T \\
\hline
\end{tabular}

augmented systemic inflammation and innate immune responses, at least partly by increasing the number of leukocytes and platelets as well as by promoting pro-inflammatory factor secretion.

In the pathophysiology of atherosclerosis, circulating modified lipoproteins are deposited on atheroma-prone arteries. Immune responses involving leukocytes and platelets have evolved to eliminate these foreign materials by initiating acute inflammatory reactions. However, such inflammation may become persistent and chronic under sustained atherogenic stimulus $[10,21]$. We

Table IV. Reagent information

\begin{tabular}{|lcc|}
\hline Name & Company & Cat. No \\
\hline Galectin-3 & Santa Cruz Biotechnology & sc-53127 \\
\hline$\alpha$-Actin & Santa Cruz Biotechnology & sc-7210 \\
\hline TB Green Prenixex Taq II & TaKaRa Biotechnology & RR820A \\
\hline RNAiso Plus & TaKaRa Biotechnology & RP047A \\
\hline Prime Script RT reagent Kit & TaKaRa Biotechnology & M170829-004b \\
\hline IL-6 ELISA Kit & NeoBioscience Biotechnology & EMC102a \\
\hline TNF- $\alpha$ ELISA Kit & NeoBioscience Biotechnology & SLBM4444V \\
\hline Oil Red O & Sigma-Aldrich & G1471 \\
\hline Sirus Red & Solarbio Biotechnology & \\
\hline
\end{tabular}


evaluated atherosclerotic areas of whole arteries by staining with Oil Red O. PM2.5 treatment increased red-positive areas (Figures $3 \mathrm{~A}, \mathrm{~B}$ ).

Under pro-inflammatory conditions, circulating leukocytes transmigrate to atherosclerotic lesions and irreversibly differentiate into macrophages [11, 22]. However, macrophages have a high degree of plasticity, generally divided into two phenotypes. The first, classically activated macrophages (M1), synthesize abundant reactive oxygen species as well as pro-inflammatory cytokines. NADPH oxidases, especially nox2, generate superoxide in monocytes, producing ROS, thereby acting as a primary cause of atheroma. These stimulate leukocyte recruitment, activation, differentiation and survival. Among these, p22phox, p47phox, TNF- $\alpha$, IL-6, IL-1 $\beta$ and IFN- $\alpha$ are associated with atherosclerosis [8, $10,11,23]$. Macrophages of the second phenotype, alternatively activated macrophages (M2), secrete anti-inflammatory factors, including IL-10, TGF- $\beta$, arginase- 1 and CD206, all of which exert anti-atherogenic effects $[10,11,22,24]$. Therefore, the ratio of $M 1$ to $M 2$ macrophages in plaques is critical for development of atherosclerosis. Fibrous caps, mainly composed of collagen, maintain plaque integrity and prevent exposure of thrombotic necrotic cores to the circulation. SMCs are the major cellular component responsible for the synthesis of extracellular matrix [25], mainly collagen, and for maintaining rigid intra-plaque structures resistant to blood pressure [26-28]. M2 macrophages contribute to the proliferation and differentiation of medial SMCs and bone marrow fibrocytes. We found that the nox2-derived ROS sources p22phox and p47phox (Figures $5 \mathrm{Aa}$ and $\mathrm{b}$ ) and pro-inflammatory factors IL- 6 and TNF- $\alpha$, as well as M1-associated cytokines iNOS and IL-12 (Figures $5 \mathrm{Ba}$ and b), were substantially elevated in the arteries of apoE $\mathrm{E}^{-/-}$mice treated with PM2.5. There was a simultaneous decrease in the M2-specific markers arginase- 1 and CD206 (Figures 5 B), indicating amplified pro-inflammatory effects of PM2.5 in atherosclerosis. The suppression of M2-switching could be at least partially explained by reduced SMCS and collagen content in atherosclerotic plaques of aortic roots (Figures $4 \mathrm{BC}$ and $\mathrm{d}$ ).

In conclusion, we demonstrated the impact of PM2.5 exposure on the progression of atherosclerosis in a classical mouse model of cardiovascular disease. Our evidence suggested elevated amounts of platelets, increased inflammation and skewing toward a M1 subtype, being possible mechanisms of destabilization of atherosclerotic plaques. However, one should consider that the concentration of PM2.5 and the route of PM2.5 ingestion were different from real-life situations. This study was an animal study attempting to maximally imitate the harmful effects of PM2.5 on organisms.

\section{Acknowledgments}

Xuecan Zhu and Pei Zhao contributed equally to this work.

This study was supported by funding from the Hebei provincial government funded clinical talent training and basic research project plan of 2015.

\section{Conflict of interest}

The authors declare no conflict of interest.

\section{References}

1. Huo Y, Schober A, Forlow SB, et al. Circulating activated platelets exacerbate atherosclerosis in mice deficient in apolipoprotein E. Nat Med 2003; 9: 61-7.

2. Li Q, Liu H, Alattar M, et al. The preferential accumulation of heavy metals in different tissues following frequent respiratory exposure to PM2.5 in rats. Sci Rep 2015; 5: 16936.

3. Kral JB, Schrottmaier WC, Salzmann M, Assinger A. Platelet interaction with innate immune cells. Transfus Med Hemother 2016; 43: 78-88.

4. Gu XY, Chu X, Zeng XL, Bao HR, Liu XJ. Effects of PM2.5 exposure on the Notch signaling pathway and immune imbalance in chronic obstructive pulmonary disease. Environ Pollut 2017; 226: 163-73.

5. Gai HF, An JX, Qian XY, Wei YJ, Williams JP, Gao GL. Ovarian damages produced by aerosolized fine particulate matter (PM2.5) pollution in mice: possible protective medications and mechanisms. Chin Med J (Engl) 2017; 130: 1400-10.

6. Fang L, Moore XL, Dart AM, Wang LM. Systemic inflammatory response following acute myocardial infarction. J Geriatr Cardiol 2015; 12: 305-12.

7. Ivanova EA, Orekhov AN. Monocyte activation in immunopathology: cellular test for development of diagnostics and therapy. J Immunol Res 2016; 2016: 4789279.

8. Atehortúa L, Rojas M, Vásquez GM, Castaño D. Endothelial alterations in systemic lupus erythematosus and rheumatoid arthritis: potential effect of monocyte interaction. Mediators Inflamm 2017; 2017: 9680729.

9. Stocker TJ, Ishikawa-Ankerhold H, Massberg S, Schulz C. Small but mighty: platelets as central effectors of host defense. Thromb Haemost 2017; 117: 651-61.

10. Tang CY, Mauro C. Similarities in the metabolic reprogramming of immune system and endothelium. Front Immunol 2017; 8: 837.

11. Rojas J, Salazar J, Martínez MS, et al. Macrophage heterogeneity and plasticity: impact of macrophage biomarkers on atherosclerosis. Scientifica (Cairo) 2015; 2015: 851252.

12. Walski M, Chlopicki S, Celary-Walska R, FrontczakBaniewicz M. Ultrastructural alterations of endothelium covering advanced atherosclerotic plaque in human carotid artery visualized by scanning electron microscope. J Physiol Pharmacol 2002; 53: 713-23.

13. Nathan L, Pervin S, Singh R, Rosenfeld M, Chaudhuri G. Estradiol inhibits leukocyte adhesion and transendothelial migration in rabbits in vivo: possible mechanisms for gender differences in atherosclerosis. Circ Res 1999; 85: 377-85.

14. Meng X, Zhang Y, Yang KQ, Yang YK, Zhou XL. Potential harmful effects of PM2.5 on occurrence and progression of acute coronary syndrome: epidemiology, mecha- 
nisms, and prevention measures. Int J Environ Res Public Health 2016; 13: pii: E748.

15. Solenkova NV, Newman JD, Berger JS, Thurston G, Hochman JS, Lamas GA. Metal pollutants and cardiovascular disease: mechanisms and consequences of exposure. Am Heart J 2014; 168: 812-22.

16. Ozcicek A, Ozcicek F, Yildiz G, et al. Neutrophil-to-lymphocyte ratio as possible indicator of epicardial adipose tissue in patients undergoing hemodialysis. Arch Med Sci 2017; 13: 118-23.

17. Ellulu MS, Patimah I, Khaza'ai H, Rahmat A, Abed Y. Obesity and inflammation: the linking mechanism and the complications. Arch Med Sci 2017; 13: 851-63.

18. Pei Y, Jiang R, Zou Y, et al. Effects of fine particulate matter (PM2.5) on systemic oxidative stress and cardiac function in ApoE-/- mice. Int J Environ Res Public Health 2016; 13: pii: E484.

19. Sorci-Thomas MG, Thomas MI. Microdomains, inflammation and atherosclerosis. Circ Res 2016; 118: 679-91.

20. Kzhyshkowska J, Gudima A, Moganti K, Gratchev A, Orek hov A. Perspectives for monocyte/macrophage-based diagnostics of chronic inflammation. Transfus Med Hemother 2016; 43: 66-77.

21. Wu MY, Li CJ, Hou MF, Chu PY. New insights into the role of inflammation in the pathogenesis of atherosclerosis. Int J Mol Sci 2017; 18: 2034.

22. Peled M, Fisher EA. Dynamic aspects of macrophage polarization during atherosclerosis progression and regression. Front Immunol 2014; 5: 579.

23. Medbury HJ, Williams H, Fletcher JP. Clinical significance of macrophage phenotypes in cardiovascular disease. Clin Transl Med 2014; 3: 63.

24. Mills CD. Anatomy of a discovery: M1 and M2 macrophages. Front Immunol 2015; 6: 212.

25. Linares A, Perales S, Palomino-Morales RJ, Castillo $M$, Alejandre MJ. Nutritional control, gene regulation, and transformation of vascular smooth muscle cells in atherosclerosis. Cardiovasc Hematol Disord Drug Targets 2006; 6: 151-68.

26. Beamish JA, He P, Kottke-Marchant K, Marchant RE. Molecular regulation of contractile smooth muscle cell phenotype: implications for vascular tissue engineering. Tissue Eng Part B Rev 2010; 16: 467-91.

27. Raines EW. The extracellular matrix can regulate vascular cell migration, proliferation, and survival: relationships to vascular disease. Int J Exp Pathol 2000; 81: 173-82

28. Cheng JK, Wagenseil JE. Extracellular matrix and the mechanics of large artery development. Biomech Model Mechanobiol 2012; 11: 1169-86. 